\title{
Resultados de un Programa de Deshabituación Tabáquica en Atención Primaria
}

\author{
S. Garrido Elustondo, V. Hurtado Riesgo ${ }^{1}$, E.Vicente Maríaª I. González \\ RomO ${ }^{3}$, Ma C. SeRrano FERnÁnDEZ $Z^{4}$ \\ M édico. Especialista en Mediana Preventiva y Salud Pública. Unidad de Forma -

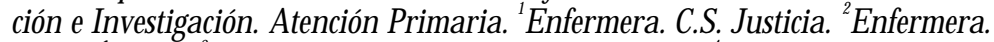 \\ C.S. Águilas. ${ }^{3}$ Enfermera. C.S. Paseo de Extremadura. ${ }^{4}$ Enfermera. C.S. Valle \\ Inclán. Área 7. Madrid
}

\section{RESUMEN}

Fundamento: la intervención de los profesiona les sanitarios de Atención Primaria en la deshabi tuación tabáquica se recomienda desde diferentes instituciones sanitarias.

Objetivos: conocer el porcentaje de individuos que dejan de fumar al finalizar un taller de deshabituación tabáquica y a los 3, 6 y 12 meses. Cuan tificar la disminución en el número de cigarrillos fumados, y analizar variables predictores de des habituación tabáquica.

Material y métodos: población de estudio: todos los participantes en los talleres desarrollados des de enero de 1999 a mayo de 2000. Los talleres constan de 6 sesiones de 90 minutos de duración cada una. Variables estudiadas: edad, sexo, $n^{o}$ de cigarrillos inicialmente fumados, cigarrillos fuma dos al finalizar el taller y a los 3, 6 y 12 meses.

Resultados: participantes: 143, 63 mujeres y 80 varones. Edad: media 50, desviación estándar (DE) 12,7, cigarrillos al inicio: media 25, DE 13,5.

Abandonan el taller 44 participantes, $(30,8 \%)$. Entre los 99 que terminan el taller, no fuman 62 $(62,6 \%)$ al finalizar el taller y 33 (33,3\%), 29 (29,3\%), y $25(27,5 \%)$ a los 3, 6 y 12 meses. La dis minución media en el número de cigarrillos fumados es al final del taller y a los 3, 6 y 12 meses de 20,2, 14,4, 11,5 y 11, respectivamente. En los hombres, la edad actúa como variable predictora de deshabitua ción odds ratio (OR) 1,02 (1-1,03) $p=0,04$.
Results of Smoking Cessation Program in Primary Health Care

\section{ABSTRACT}

Background: the intervention of health workers in smoking cessation is recomended by different health organizations.

Objectives: to study the percentage of partici pans that stop smoking finished program and 3, 6 and 12 months later, to count the decrease of the daily cigarettes smoked and study the predicting factors of smoking cessation

Material and methods: patients: all the partici pants of groups developed since February 1999 to May 2000. The intervention consist in six sesions of 90 minutes each one. Variables for each partici pant: sex, age, daily number of cigarettes, number of cigarettes measured at finished intervention and after 3 months, 6 months and a year.

Results: number of participants: 143, 80 males and 63 women. Age: mean 50, SD 12.7, number of daily cigarettes: mean $25, S D$ 13.5. Give up on the intervention 44, $30.8 \%$ of participants. Analizing only who finished the intervention, stopped smo king $62(62.6 \%)$ after intervention and $33(33.3 \%)$, $29(29.3 \%)$, and $25(27.5 \%)$ at 3, 6, and 12 months. The mean of the decreased of the number of cigarettes is 20.2, 14.4, 11.5, and 11 finished the in tervention and after 3, 6, and 12 months. In males, age acts as predicting factor of smoking cessation OR $1.02(1-1.03) p=0.4$.

Conclusions: the percentage of participants who stop smoking is satisfactory although the number of 
Conclusiones: el porcentaje de participantes que dejan de fumar es satisfactorio, pero el número de abandonos del taller es alto. Nos planteamos establecer estrategias destinadas a reforzar la adherencia a los talleres y a mejorar su efectividad.

Palabras clave: Deshabituación tabáquica. In tervención. Educación grupal. Atención Primaria. desertions is high. We are planning to established es trategies in order to decreased the desertions and improve the results of the intervention.

Key words: Smoking cessation. Intervention. Group education. Primary Health Care.

\section{INTRODUCCIÓN}

El consumo de tabaco es la principal causa prevenible de enfermedad y muerte en los países desarrollados. En España, se estima que es el responsable del $15 \%$ del total de muertes y del $10 \%$ del número total de años de vida perdidos ${ }^{1}$. La prevalencia del tabaquismo, a pesar de haber sufrido un ligero descenso, sigue siendo alta, y según datos de la Encuesta Nacional de Salud de 1997, se estima que fuma el $35,7 \%$ de los mayores de 16 años, 44,8\% de los hombres y $27,2 \%$ de las mujeres ${ }^{2}$.

Reducir la prevalencia del tabaquismo es un objetivo de Salud Pública y los servicios de Atención Primaria constituyen un lugar idóneo para la ejecución de las posibles estrategias. Así, la intervención activa de los profesionales sanitarios de Atención Primaria en la deshabituación tabáquica se recomienda desde diversos grupos de expertos e instituciones sanitarias: US Service Task Force, Canadian Task Force ${ }^{4}$, PAPPS Agency for Health Care Policy and Research $^{6}$. La OMS, como recoge el Convenio Marco sobre la Lucha Antitabaco, lo considera una línea prioritaria de actuación.

Existen protocolizados diferentes métodos para ayudar a dejar de fumar: desde la intervención mínima realizada en la consulta tanto por médicos como por enfermeras, a intervenciones más intensivas. Estas intervenciones han sido evaluadas, obteniendo diferentes resultados según el tipo de actividad y la población a la que van dirigidas. Así, según datos publicados, el consejo médico antitabaco consigue un porcentaje de pacientes que abandonan el hábito entre el 3 y el $13 \%$, con una media de $6 \%^{7}$. Los resultados de las intervenciones más intensivas varían en función de la estructura de éstas, y en estudios no controlados presentan porcentajes entre el 13 y el $38 \%$ de pacientes que consiguen abandonar el hábito, con una media de $22 \%$ de abstinentes al año de seguimiento ${ }^{7}$.

En estudios controlados, en una reciente revisión, se concluye que los pacientes que reciben consejo breve abandonan el hábito tabáquico en un porcentaje mayor que los que no lo reciben (OR 1,69), intervalo de confianza (IC) al 95\% 1,45-1,98 ${ }^{8}$, los que reciben terapias más intensivas frente a los que no las reciben lo hacen en un porcentaje mayor (OR 2,13, IC al 95\% 1,77-2,56) ${ }^{8}$. Al analizar terapias g rupales, los que asisten a éstas abandonan el hábito tabáquico en un porcentaje mayor que los que no asisten (OR 1,91, IC al 95\% 1,20-3,04) ${ }^{9}$.

La capacidad para generalizar los resultados de estas intervenciones es difícil debido a la propia heterogeneidad de las mismas 9 . Es recomendable, por lo tanto, para conocer su impacto a nivel local evaluar los resultados de las terapias utilizadas.

En febrero de 1999 se ponen en marcha talleres de deshabituación tabáquica en 4 Centros de Salud del Área 7 de Atención Primaria de Madrid. Este estudio tiene como objetivo principal conocer el porcentaje de individuos que consiguen dejar de fumar al finalizar el taller y a los 3, 6 y 12 meses de seguimiento. Como objetivos secundarios, cuantificar la reducción en el número de cigarrillos fumados y estudiar posibles variables predictoras en el abandono del hábito tabáquico.

\section{MATERIAL Y MÉTODOS}

Realizamos un estudio descriptivo prospectivo en el que analizamos los resultados recogidos de todos los participantes a los talleres que tienen lugar entre febrero de 1999 hasta mayo de 2000, para alcanzar un tamaño mínimo muestral de 135 participantes ( $p=0,22$; error máximo de precisión $\pm 7 \%$, nivel de confianza del 95\%). Los participantes en este estudio se captaron de forma consecutiva, en la medida en que realizan los talleres y se distribuyen de forma similar en los 4 Centros, que son semejantes en cuanto a número de profesionales y población adscrita.

La captación a los talleres se realiza a través de las consultas del médico o la enfermera y de carteles informativos colocados en los Centros. A los interesados se les realiza una entrevista, sesión previa, en la que se recogen datos personales: edad, sexo y antecedentes personales orientados a descartar los siguientes criterios de exclusión: menor de 18 años, patología psiquiátrica grave (depresión mayor, psicosis), discapacidad visual o auditiva, analfabetismo funcional, toxicomanías asociadas graves (dependencia a opiáceos, alcohol y otros), tal y como recomiendan los programas de deshabituación tabáquica, con el fin de favorecer la comprensión de las instrucciones del programa y el beneficio de la intervención. No se considera un factor de exclusión haber participado 
previamente en un grupo de este tipo. Tras la entrevista, y si no constituyen un motivo de exclusión, se organizan grupos de 10 personas de forma consecutiva, sin tener en cuenta a la hora de formar los grupos factores como la edad y sexo, y posteriormente se notifica la fecha de inicio del taller.

Los talleres se realizan en el Centro de Salud, y en cada Centro son impartidos por el mismo profesional. Estos profesionales recibieron una formación específica, y consensuaron la metodología a emplear en el taller. El objetivo del taller es conseguir la deshabituación tabáquica. Cada taller se articula en 6 sesiones de 90 minutos de duración cada una. Sus contenidos y objetivos de forma resumida se presentan en la figura 1.

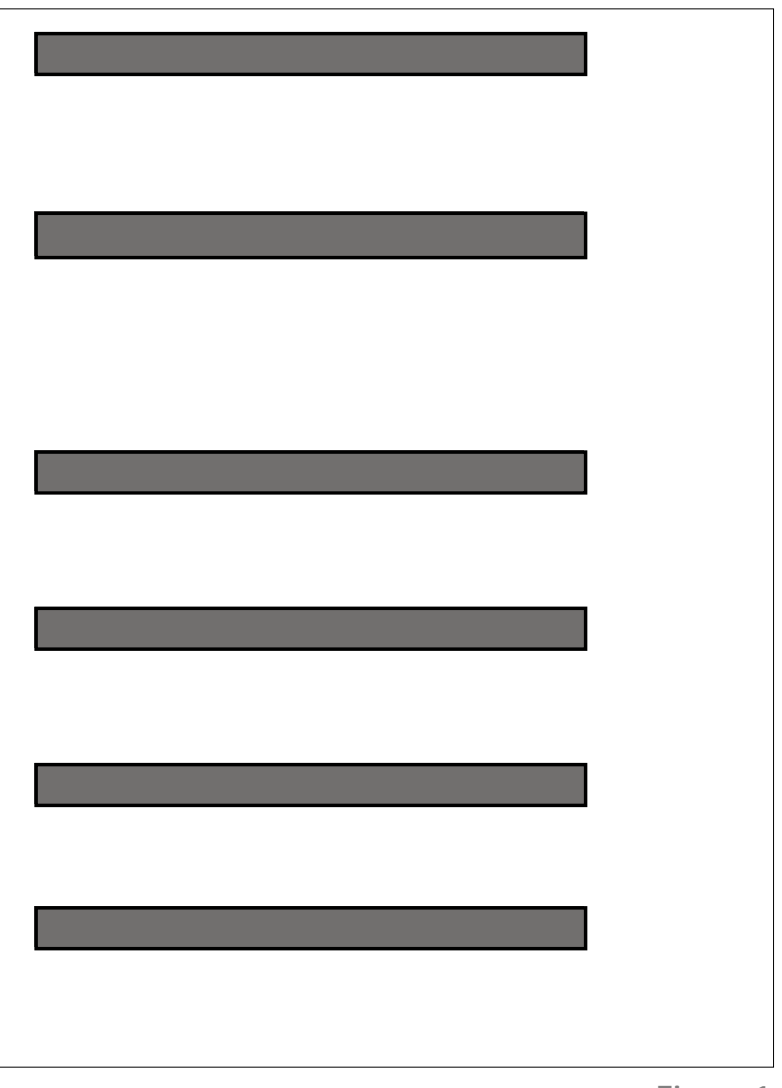

Estructura de los talleres de deshabituación tabáquica.

Para cada participante en los talleres se recogen las siguientes variables: edad, sexo, $\mathrm{n}^{\circ}$ de cigarrillos al inicio del taller. Al finalizar el taller se cuantifica el número de cigarrillos que fuman, y se les realiza un seguimiento para conocer el número de cigarrillos que fuman a los 3, 6 y 12 meses de terminar el taller, como recomiendan diferentes guías de práctica clínica en deshabituación tabáquica ${ }^{10}$. El seguimiento se realiza telefónicamente por el mismo profesional que ha impartido los talleres. A los que no finalizan el taller, ya no se les realiza seguimiento alguno. Se considera que han logrado abandonar el hábito tabáquico cuando al año de seguimiento se mantienen en 0 cigarrillos. Se asume que el número de cigarrillos que fuman es el que ellos refieren, no realizándose ninguna otra prueba para verificar el dato.

El procesamiento y análisis de los datos se realizó mediante el programa SPSS v.10, calculándose medias y DE en el caso de variables cuantitativas, frecuencias absolutas y relativas para las cualitativas, Chi cuadrado para la comparación de proporciones y t de Student para la comparación de medias ( $\mathrm{t}$ de Student de datos apareados para la diferencia en el número de cigarrillos fumados a lo largo del seguimiento). Para conocer las posibles variables predictoras del abandono del hábito tabáquico se empleó el análisis de regresión logística con el método Forward stepwise, siendo la variable dependiente el abandono del tabaco al año de seguimiento, e introduciendo en el modelo las variables edad, sexo y número de cigarrillos iniciales.

\section{RESULTADOS}

Se han estudiado 143 participantes, 63 mujeres $(44 \%)$ y 80 varones $(56 \%)$. La media $(\mathrm{m})$ de edad es de 50 años, con una DE de 13,5, y un rango que va de 24 años a 80 . El número de cigarrillos que fuman al inicio de los talleres tiene un promedio de 25 cigarrillos, una DE de 12,7 y un rango que va de 3 a 60 .

Abandonan el taller (no finalizan las 6 sesiones) 44 personas, el 30,8\%. En la tabla I se muestran las características de las variables estudiadas en los participantes que abandonan y los que no. No se encuentran diferencias estadísticamente significativas en ambos grupos.

Contabilizando únicamente a los que terminan el taller, 99 participantes $(69,2 \%)$, al finalizar el mismo han conseguido dejar de fumar 62, el 62,6\% (IC al 95\% 52,3-71,9). Se mantienen en 0 cigarrillos a los 3,6 y

\begin{tabular}{|c|c|c|c|}
\hline \multicolumn{4}{|c|}{$\begin{array}{c}\text { CARACTERISTIICAS SEGÚN LAS VARIABLES ESTUDIADAS } \\
\text { DE LOS INDIVIDUOS QUE ABANDONAN EL TALLER } \\
\text { Y LOS QUE NO LO ABANDONAN }\end{array}$} \\
\hline Variables & $\begin{array}{l}\text { Abandonan } \\
(n=44)\end{array}$ & $\begin{array}{l}\text { No abandonan } \\
(\mathrm{n}=99)\end{array}$ & $p$ \\
\hline Hombres & $28(63,3 \%)$ & $52(52,5 \%)$ & NS \\
\hline Mujeres & $16(36,4 \%)$ & $47(47,5 \%)$ & NS \\
\hline $\begin{array}{l}\text { № de cigarrillos } \\
\text { que fuman al inicio } \\
\text { del taller (media } \pm D E \text { ) }\end{array}$ & $26,57 \pm 14,18$ & $24,68 \pm 11,98$ & NS \\
\hline $\begin{array}{l}\text { Edad } \\
\text { (media } \pm D E)\end{array}$ & $52,09 \pm 11,45$ & $49,99 \pm 13,69$ & NS \\
\hline
\end{tabular}


12 meses de finalizar el taller 33, el 33,3\% (IC al 95\% 24,9-44,4); 29, el 29,3\% (IC al 95\% 21,2-40,2); y 25, el $27,5 \%$ (IC al 95\% 18,9-37,9) respectivamente, considerando a aquellos con los que conseguimos contactar, el $100 \%$ a los 3 y 6 meses y el $91 \%$ a los 12 meses (Fig. 2). En la figura 3 se presenta el porcentaje de individuos que dejan de fumar, considerando los 143 individuos que comienzan el taller: 43,3\% (IC al 95\% 37,854,6), 23,1\% (IC al 95\% 18,5-33,2), 20,3\% (IC al 95\% 16,6-31) y 19,1 (IC al 95\% 12,4-25,7), al finalizar el taller y a los 3, 6 y 12 meses respectivamente.

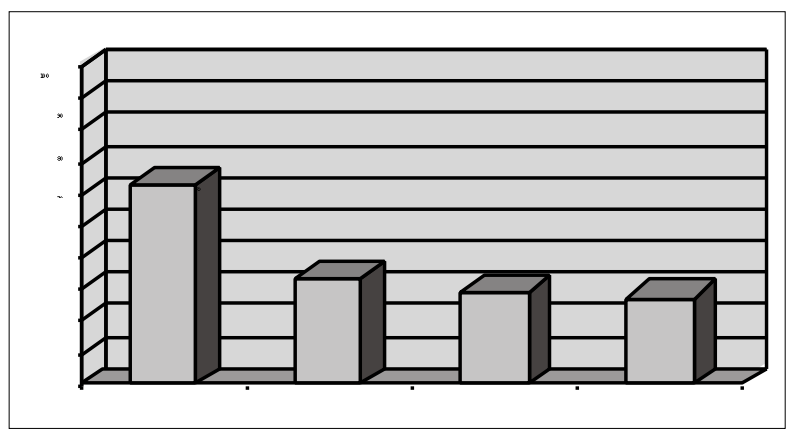

Figura 2

Porcentaje de individuos que dejan de fumar a lo largo del seguimiento. Teniendo en cuenta únicamente los que finalizan el taller $(n=90)$.

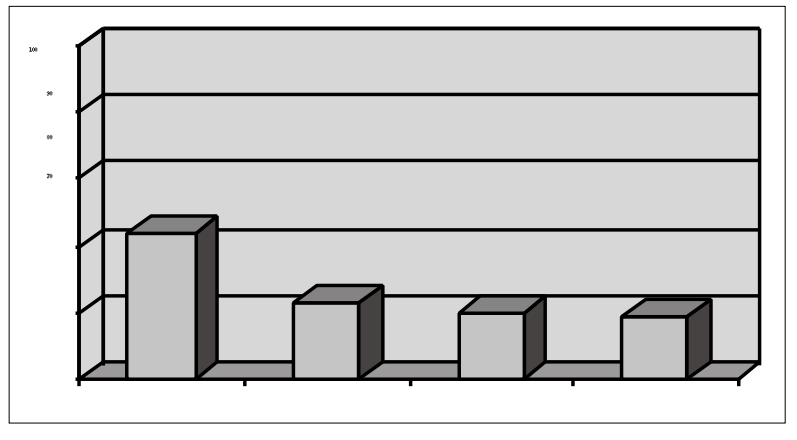

Figura 3

Porcentaje de individuos que dejan de fumar a lo largo del seguimiento. Teniendo en cuenta todos los que comienzan los talleres $(n=143)$.
En los 99 sujetos que no abandonan los talleres, la media y desviación estándar (DE) de los cigarrillos fumados al finalizar el taller y a los 3, 6 y 12 meses es: $5,4(3,8-7,0)$, DE 9,2; 11,3 (9,0-13,5), DE 12,6; 14,0 (11,5-16,5), DE 14,2; 15,1 (12,6-17,7), DE 13,5 respectivamente. La disminución media de los cigarrillos fumados a lo largo del seguimiento respecto a los fumados al inicio del taller se presenta en la tabla II.

El resultado del análisis de regresión logística se presenta en la tabla III. Encontramos una interacción en la edad y el sexo. En los hombres, la edad actúa como un factor pronóstico para el abandono del hábito tabáquico $\mathrm{OR}=1,02$ por cada año, (IC al 95\% 1$1,033) \mathrm{p}=0,04$.

\section{DISCUSIÓN}

Este estudio se ha realizado analizando los resultados de todos los participantes a los talleres, captados de forma consecutiva desde que éstos comenzaron hasta alcanzar el tamaño muestral requerido. El grupo seleccionado para el estudio representa a la población que participa en los talleres impartidos en estos centros. No se ha estudiado si el acceso al taller es a propuesta del médico o a demanda, hecho que según otros estudios aporta diferencias en el resulta$\mathrm{do}^{11}$. La forma de realizar la captación, que se realiza en el Centro de Salud, hace que los resultados sean representativos de individuos que acuden a los Centros de Salud.

Otra limitación de este estudio es no haber considerado un factor de exclusión haber participado previamente en un grupo de este tipo.

El porcentaje de abandonos parece alto: $30,8 \%$. Es difícil compararlos con otros programas, ya que la duración de los mismos es diferente. Entre las posibles razones, encontramos que el programa va dirigido a la población general, que acude a los Centros de Salud. Se ha observado que en programas dirigidos a individuos afectados con determinadas patologías: infarto agudo de miocardio, ingresados en servicios de neumología, los porcentajes de abandono son menores $^{12,13}$.

\section{Tabla II}

DIFERENCIA EN EL NÚMERO DE CIGARRILLOS FUMADOS A LO LARGO DEL SEGUIMIENTO RESPECTO A LOS FUMADOS AL INICIO

\begin{tabular}{lcccc}
\hline & Al finalizar el taller & A los 3 meses & A los 6 meses & A los 12 meses \\
\hline $\begin{array}{l}\text { Diferencias } \\
\text { relacionadas } \\
\text { (media } \pm D E)\end{array}$ & $20,17 \pm 13,48$ & $14,40 \pm 14,30$ & $11,52 \pm 12,61$ & $11,03 \pm 12,83$ \\
$\begin{array}{l}\text { IC al } 95 \% \text { para } \\
\text { la diferencia }\end{array}$ & $(17,83-22,51)$ & $(11,87-16,93)$ & $(9,29-13,75)$ & $(8,69-13,38)$ \\
$p$ & $<0,001$ & $<0,001$ & $<0,001$ & $<0,001$ \\
\hline
\end{tabular}

DE: desviación estándar; IC: intervalo de confianza; p: significación estadística. 
Tabla III

ANÁLISIS DE REGRESIÓN LOGISTICA DE LA VARIEBLES PREDICTORAS DEL ABANDONO DEL TABACO

\begin{tabular}{lccccc} 
Variable & B & Wald & $p$ & R & OR (IC al 95\%) \\
\hline Sexo y edad & 0,02 & 3,983 & 0,04 & $-0,774$ & $1,017(1-1,033)$
\end{tabular}

p: significación estadística; I.C.: intervalo de confianza. Variables que no están en la ecuación: edad (p: 0,443); sexo (p: 0,620); cigarrillos inicial $(p: 0,741)$.

El porcentaje de éxito (fumar 0 cigarrillos) al finalizar el taller entre los que no lo abandonan es alto: $62,6 \%$, pero si tenemos en cuenta todos los que comienzan el taller disminuye considerablemente: $43,3 \%$, y es algo más baja que en intervenciones similares ${ }^{14}$. Este porcentaje decae a lo largo del seguimiento, de manera similar a como se observa en otros estudios $^{14-18}$. A su vez, al cuantificar la diferencia en el número de cigarrillos fumados con respecto a los fumados al iniciar el taller, ésta se hace cada vez menor a lo largo del seguimiento. Ambos hechos confirman la gran frecuencia con la que se producen incrementos en el número de cigarrillos fumados a lo largo del tiempo, y apoya la conveniencia de realizar un seguimiento a los participantes a los talleres para conocer realmente los resultados.

En este estudio, no nos planteamos seguir a los pacientes que abandonaron los talleres, pero en un futuro nos parece una medida de interés, ya que en ocasiones una intervención breve es suficiente para inducir un abandono del hábito.

Por otro lado, el mantenimiento de las abstinencias es un aspecto de gran interés, siendo aconsejable estudiar factores relacionados con las recaídas.

En cuanto al porcentaje de participantes que al año de seguimiento siguen fumando 0 cigarrillos, en nuestro estudio es de 19,1, cifra que encontrándose dentro del intervalo de resultados publicados, (13 y $38 \%)^{7}$ está por debajo de la mediana $(22,5)$. Las razones de este hecho pueden deberse al alto porcentaje de abandonos (si tenemos en cuenta sólo a los que terminan el taller, el porcentaje de abstinentes al año de seguimiento es de $27,5 \%$ ).

En relación a las variables predictoras, en nuestro estudio el ser hombre y la edad interactúan como predictores en la deshabituación tabáquica. En otros estudios, el sexo (hombre) ${ }^{19}$ y la edad ${ }^{19-22}$ actúan como variables predictoras por separado. No encontramos que el número de cigarrillos fumados inicialmente actúe como predictor, hecho que se observa en otros estudios $^{16,20,22}$. Hemos de tener en cuenta que el cálculo de las variables predictoras no es objetivo principal de este estudio, y posiblemente haga falta un tamaño de muestra mayor. Por otro lado, intuimos que otras variables que no se han tenido en cuenta en este estudio: (patología crónica previa, grado de dependencia tabáquica, motivación para abandonar el hábito al inicio del grupo) podrían actuar como predictoras, por lo que sería necesario otro estudio con este objetivo principal para demostrarlo.
Otra limitación de nuestro estudio es que no se verifica el abandono del tabaco midiendo el CO espirado u otros parámetros biológicos, al no disponer en los Centros de Salud donde se realizan los talleres de Cooxímetros, por lo que habría que aplicar una corrección a la baja.

Los resultados de los talleres, como actividad preventiva, nos parecen satisfactorios. No obstante, el coste de estos talleres en recursos humanos es importante, tanto por la duración del taller: 6 sesiones de 90 minutos de duración cada uno como por el tiempo invertido en realizar el seguimiento establecido: contacto a los 3, 6 y 12 meses, por lo que debemos procurar optimizar los recursos. Así, nos planteamos en adelante utilizar el test de Richmond en la entrevista previa a la inclusión de los individuos en los talleres. Hay trabajos que han demostrado el valor de tests de motivación (tipo Richmond) como buenos predictores de la cesación tabáquica ${ }^{20,23}$ aconsejando su uso como herramienta que permita detectar a aquellos participantes con una menor probabilidad de éxito ${ }^{20}$. Pretendemos detectar a aquellos individuos menos motivados y reforzar en ellos intervenciones que permitan avanzar en su proceso de motivación, para así, por un lado disminuir el número de abandonos del taller y por otro conseguir entre los asistentes a los talleres un mayor porcentaje de individuos que dejan de fumar. Asumiendo, aún así, que algunos participantes abandonarán el taller, nos planteamos grupos iniciales de 12 personas, en lugar de 10.

Nos parece pertinente seguir evaluando estos talleres periódicamente, para conocer su impacto a nivel local e ir introduciendo las medidas correctoras necesarias.

\section{AGRADECIMIENTOS}

Nuestro agradecimiento a Ricardo Ruiz de Adana Pérez y a Pedro Nogales Aguado por sus valiosas recomendaciones metodológicas y sugerencias al artículo.

\section{CORRESPONDENCIA:}

Sofía Garrido Elustondo

Unidad de Formación. Gerencia Área 7. INSALUD

C/ Espronceda, 24-4 ${ }^{\mathrm{a}} \mathrm{pl}$.

28003 Madrid

e-mail: sgarrido@gapm07.insalud.es 


\section{Bibliografía}

1. González J, Rodríguez F, Martín JM, Banegas JR. Muertes atribuibles al consumo del tabaco en España. Med Clin (Barc) 1989; 92: 15-8.

2. Encuesta Nacional de Salud de España. 1997. Madrid: Ministerio de Sanidad y Consumo. Madrid, 1999.

3. U.S. Preventive Services Task Force. Guide to clinical preventive services. $2^{\mathrm{a}}$ ed. Baltimore: Williams and Wilkins, 1996. p. 163-75.

4. Canadian Task Force on the periodic health Examination. The periodic health examination 1985 update. Can Med Assoc J 1986; 134: 724-7.

5. Sociedad Española de Medicina Familiar y Comunitaria. Programa de Actividades Preventivas y Promoción de la Salud (PAPPS). Actualización 2001. Aten Prim 2001; 28 (Supl. 1): 224.

6. Agency for Health Care Policy and Research. Smoking cessation. Clinical Practice Guidelines. JAMA 1996; 275: 1270-80.

7. Schwartz JL. Methods of smoking cessation. Med Clin North Am 1992; 76: 451-76.

8. Silagy C. Physician advice for smoking cessation (Cochrane Review). The Cochrane Library Issue 3. Oxford: Update Software, 2000.

9. Stead LF, Lancaster T. Group behaviour therapy progammes for smoking cessation (Cochrane Review). The Cochrane Library, Issue 3. Oxford: Update Software, 2000.

10. A clinical practice guideline for treating tobacco use and dependence: A US Public Health Service report. The Tobacco Use and Dependence Clinical Practice Guideline Panel, Staff, and Consortium Representatives. JAMA 2000; 283: 3244-54.

11. Torrecilla M, Barrueco M, Jiménez CA, Maderuelo JA, Plaza MD, Hernández MA. El médico y el paciente en la toma de decisión del abandono del tabaco. Repercusión de la iniciativa en el resultado de la intervención. Arch Bronconeumol 2001; 37: 127-34.

12. Moreno A, Ochoa FJ, Ramalce E, Saralegui I, Fernández MV, Quintana M. Eficacia de una intervención para dejar de fumar en pacientes con infarto de miocardio. Med Clin (Barc) 2000; 114: 209-10.

13. Jiménez CA, González JM, Escudero S, Pérez A, García A, Florez S. Resultados de un programa de deshabituación tabáquica en una Unidad de Neumología y Cirugía Torácica en España. Arch Bronconeumol 1996; 32: 138-41.

14. Aleixandre E, Casanova MA, Mitjans J, Sánchez J, Sanmartín A. Ensayo clínico de dos intervenciones de deshabituación tabáquica en atención primaria. Aten Prim 1998; 22: 424-8.

15. Comas A, Gutiérrez R, López L, Cueto E. Efectividad a largo plazo del conseso antitabaco en atención primaria: El proceso de recaída. Rev Esp Salud Pública 1996; 70: 283-93.

16. Martín C, García R, Jane C, Nebot M, Galán S, Aliaga M, et al. Evaluación a medio plazo de un programa de ayuda a los fumadores. Med Clin (Barc) 1997; 109: 744-8.

17. Torrecilla M, Barrueco M, Maderuelo JA, Jiménez CA, Plaza MD, Hernández MA. Deshabituación tabáquica en una consulta de atención primaria: eficacia del consejo médico, la intervención mínima y la terapia sustitutiva con nicotina al año de seguimiento. Aten Prim 2001; 27: 629-36.

18. Comas A, Suárez R, López ML, Cueto A. Coste - efectividad del consejo sanitario antitabaco en atención primaria de salud. Gac Sanit 1998; 12: 126-32.

19. Moragues I, Nebot M, Muñoz M, Ballestin M, Salto E. Evaluación de un programa de intervención comunitaria de deshabituación tabáquica. Gac Sanit 1999;13: 456-61.

20. Córdoba R, Martín C, Casas R, Barbera C, Botaya M, Hernández $\mathrm{A}$, et al. Valor de los cuestionarios breves en la predicción del abandono del tabaco en atención primaria. Aten Prim 2000; 24: 74-82.

21. García A, Hernández I, Álvarez D. El abandono del hábito tabáquico en España. Med Clin (Barc) 1991; 97: 161-5.

22. Torrecilla M, Barrueco M, Maderuelo JA, Jiménez CA, Plaza MD, Hernández MA. ¿Se puede predecir el éxito de la intervención mínima tabaquismo? Prev Tab 2001; 3: 13-9.

23. Physicians urged to promote smoking cessation aids MMWR 2000; 49: 665-9. 